\title{
DEFERRED CORRECTION FOR THE INTEGRAL EQUATION EIGENVALUE PROBLEM
}

\author{
K. W. CHU and A. SPENCE
}

(Received 19 August 1980)

(Revised 21 November 1980)

\begin{abstract}
This paper considers the improvement of approximate eigenvalues and eigenfunctions of integral equations using the method of deferred correction. A convergence theorem is proved and a numerical example illustrating the theory is given.
\end{abstract}

\section{Introduction}

Consider the linear integral equation

$$
\lambda x(s)=\int_{a}^{b} k(s, t) x(t) d t
$$

where $a$ and $b$ are finite, the kernel $k(s, t)$ is known, and $\lambda$ and $x(s)$ are the unknown eigenvalue and associated eigenfunction. We do not assume that $k(s, t)$ is symmetric. In operator notation (1.1) can be written as

$$
\lambda x=K x
$$

and we assume that $K$ is a compact integral operator on $C[a, b]$. A survey of some basic results from functional analysis, which includes a discussion of compact integral operators, is given in part $I$ of [3]. There it is shown that if $k \in C([a, b] \times[a, b])$ then $K$ is compact on $C[a, b]$. Weakly singular kernels of the form $\log |s-t|$ and $|s-t|^{\alpha}, \alpha>-1$, are also shown to give rise to compact integral operators. Theoretical results about compact operators of interest to us are stated in the following theorem.

OCopyright Australian Mathematical Society 1981 
THEOREM 1. (a) The eigenvalues of $K$ form a discrete set in the complex plane with zero as the only possible limit point.

(b) Each nonzero eigenvalue has finite geometric and algebraic multiplicities.

When necessary the eigenvalues of (1.2) can be numbered as follows:

$$
\left|\lambda_{1}\right| \geqslant\left|\lambda_{2}\right|>\cdots \geqslant 0 \text {, }
$$

with

$$
\lambda_{i} x_{i}=K x_{i}
$$

To obtain approximations to $\lambda$ and $x$ a related matrix equation

$$
\nu \mathbf{u}=\mathbf{K}_{n} \mathbf{u}
$$

will be set up which can be solved by standard techniques, for example, reduction to upper Hessenberg form and then $Q R$. (See [17].) If $k \in C([a, b] \times$ $[a, b])$ then $\mathbf{K}_{n}$ can be derived as follows. Let

$$
\int_{a}^{b} y(t) d t \cong \sum_{j=1}^{n} w_{j} y\left(t_{j}\right)
$$

denote a quadrature rule with weights $w_{j}$ and nodes $t_{j}$. Equation (1.1) is approximated by

$$
\nu u(s)=\sum_{j=1}^{n} w_{j} k\left(s, t_{j}\right) u\left(t_{j}\right), \quad a<s \leqslant b,
$$

and the matrix eigenvalue problem (1.4) obtained by putting $s=t_{i}, i=$ $1, \ldots, n$, with $\left(\mathbf{K}_{n}\right)_{i j}=w_{j} k\left(t_{i}, t_{j}\right)$. For weakly singular kernels an approximate equation can be derived using product integration. (See [3], [5].)

Approximate methods for (1.1) have been well analysed in the past (see Chapter 3 of [5] and the references quoted therein) and theoretical results have been obtained. For example, to analyse the quadrature method above one can rewrite (1.6) in operator form as

$$
\nu u=K_{n}\left(r_{n} u\right)
$$

where $r_{n}$ is defined for any $f \in C[a, b]$ by

$$
r_{n} f=\left[f\left(t_{1}\right), f\left(t_{2}\right), \ldots, f\left(t_{n}\right)\right]^{T}=\mathbf{f} .
$$

Also, rewrite (1.1) as

$$
\lambda x=K_{n}\left(r_{n} x\right)+\tau(x)
$$

where

$$
\begin{aligned}
\tau(x) & =K x-K_{n}\left(r_{n} x\right) \\
& =\int_{a}^{b} k(s, t) x(t) d t-\sum_{j=1}^{n} w_{j} k\left(s, t_{j}\right) x\left(t_{j}\right) .
\end{aligned}
$$


The quantity $\tau(x)$, called the truncation or discretization error, measures the error in the approximation of the integral operator. It is proved in [2] that if $\lambda$ is simple and $n$ is large enough then there exists a simple eigenvalue $\nu$ of (1.4) with corresponding eigenvector u satisfying

$$
|\lambda-\nu|,\left\|r_{n} x-\mathbf{u}\right\|_{\infty}=O\left(\|\tau(x)\|_{\infty}\right) .
$$

Since many of the theoretical questions about convergence of numerical methods have been answered, attention is turning to the efficient calculation of accurate approximations to $\lambda$ and $x$ with the aim of developing fast and reliable computer subroutines.

It is clear from (1.10) that accurate approximations to $\lambda$ and $x$ will be obtained by solving (1.4) for large $n$. However, when $n$ is large the standard eigenvalue routines will be expensive in computer time. Also $\mathbf{K}_{n}$ will have $n$ eigenvalues $\nu_{i}, i=1, \ldots, n$ (counting multiple values), which approximate in some way the eigenvalues $\lambda_{l}$ of $K$, but it is probable that only the first $l$ eigenvalues of $K$, with $l \ll n$, will be of interest. (Recall Theorem 1 above.) Thus there will be no need to compute accurately all $n$ eigenvalues of $\mathbf{K}_{n}$.

Several approaches are possible but we shall only consider one method here, namely, the method of deferred correction. The technique is first to solve (1.4) for a small value of $n$ to obtain rough approximations $\nu$ and $\mathbf{u}$, and then attempt to improve the solutions by estimating the error terms $\lambda-\nu, r_{n} x-\mathbf{u}$ using Gregory's rule to estimate $\tau(x)$ in (1.9). This approach was first used for integral equations in [8]. The approach is often used for ordinary differential equations and a framework for general functional equations is given in [11], [16]. There are other related approaches, for example, defect correction [14], and the method of Chatelin [6]. These techniques of ten require that $k(s, t)$ satisfies certain smoothness conditions in addition to those needed for the convergence rate given by (1.10), and so we would not use such techniques on nonsmooth kernels. (See however Section 6.5 of [6].)

The plan of the paper is as follows. In Section 2 we give an analysis of the method of deferred correction for simple eigenvalues of (1.1). This section contains the main theoretical result of the paper. Section 3 contains some general remarks and Section 4 an example.

\section{Deferred correction for simple eigenvalues}

The method of deferred correction has a long history in numerical analysis as a means of improving approximate solutions of initial and boundary value problems. Early accounts of its application to integral equations are given in [8] and [10]. A detailed discussion, with numerical results, of the method for second 
kind integral equations,

$$
\lambda x-K x=f,
$$

is given in Sections 4.3.3 and 4.19 of [5]. (However see Remark 1 in Section 3.) To date there has not been a satisfactory analysis of the method applied to the eigenvalue problem and indeed the technique described in [10] suffers from serious theoretical and practical difficulties.

Throughout this section we assume:

Al. The trapezoidal rule is used to construct $\mathbf{K}_{n}$ in (1.4). Hence $h=$ $(b-a) /(n-1) ; t_{i}=a+(i-1) h ; w_{1}=w_{n}=h / 2, w_{i}=h, i=2, \ldots, n-1$.

A2. $\lambda$ is a simple eigenvalue of $K$.

A3. $k \in C^{p+2}([a, b] \times[a, b]), p \geqslant 2$, which implies $x \in C^{p+2}[a, b]$. (This is the extra smoothness requirement mentioned in the introduction.)

Under these assumptions we have

$$
\tau(x)=O\left(h^{2}\right)
$$

and, for large enough $n$, we have from (1.10)

$$
|\lambda-\nu|,\left\|r_{n} x-\mathbf{u}\right\|_{\infty}=O\left(h^{2}\right) .
$$

We note, for use later in this section, that $\tau(x)$ has the following representations. (See [5], Sections 2.13 and 4.19.) First,

$$
\begin{aligned}
\tau(x) & =\int_{a}^{b} k(s, t) x(t) d t-\sum_{j=1}^{n} w_{j} k\left(s, t_{j}\right) x\left(t_{j}\right) \\
& =\sum_{l=1}^{N} h^{2 l} \cdot \frac{B_{2 l}}{(2 l) !}\left\{\Theta_{s}^{(2 l-1)}(b)-\Theta_{s}^{(2 l-1)}(a)\right\}+O\left(h^{p+2}\right),
\end{aligned}
$$

where $\Theta_{s}(t)=k(s, t) x(t)$ and $N=p / 2$, for $p$ even, $N=(p+1) / 2$, for $p$ odd. Second the Gregory representation of $\tau(x)$ has the form

$$
\tau(x)=\delta_{s}^{p}(x)+O\left(h^{p+2}\right)
$$

with

$$
\delta_{s}^{p}(x)=h\left\{\frac{1}{12}\left(\nabla \Theta_{s}(b)-\Delta \Theta_{s}(a)\right)+\cdots+c_{p}^{*}\left(\nabla^{p} \Theta_{s}(b)+(-1)^{p} \Delta^{p} \Theta_{s}(a)\right)\right\},
$$

where the $c_{j}^{*}, j=1, \ldots, p$, are known. We write $\delta_{i}^{p}(x)=\delta_{i_{i}}^{p}(x)$ and

$$
\delta^{p}(x)=\left[\delta_{1}^{p}(x), \ldots, \delta_{n}^{p}(x)\right]^{T} .
$$

Recall that the true solutions satisfy (1.9)

$$
\lambda x(s)=\sum_{j=1}^{n} w_{j} k\left(s, t_{j}\right) x\left(t_{j}\right)+\tau(x)
$$


Put $s=t_{i}, i=1, \ldots, n$, to obtain the matrix equation

$$
\lambda \mathbf{x}=\mathbf{K}_{n} \mathbf{x}+\tau(x) .
$$

This equation can be regarded as a perturbation of (1.4) since $\tau(x)$ is, in some sense, a residual. The analysis below is a form of perturbation theory which is similar to that in [13] but is repeated here because of some slight differences and because the approach in [13] had a different aim.

Let us normaiize $u$ in (i.4) by putiing

$$
\|\mathbf{u}\|_{\infty}=1
$$

and for convenience only, we make the following assumptions:

A4. The largest component of $u$ is the first and is real, that is

$$
\mathbf{u}^{T}=\left[1, \boldsymbol{\xi}_{n-1}^{T}\right] \text {. }
$$

A5. $n$ is large enough so that $x\left(t_{1}\right) \neq 0$. (Recall (2.3).)

Since $\nu$ and $u$ are approximations to $\lambda$ and $x$ we write

$$
\lambda-\nu=\mu \text {, }
$$

and

$$
\mathbf{x}-\mathbf{u}=\mathbf{z}=\left[0, \boldsymbol{\eta}_{n-1}^{T}\right]^{T},
$$

which normalises x. From (2.3) we have

$$
\mu,\left\|\eta_{n-1}\right\|_{\infty}=O\left(h^{2}\right) .
$$

Equation (2.7) becomes

$$
(\nu+\mu)(\mathbf{u}+\mathbf{z})=\mathbf{K}_{n}(\mathbf{u}+\mathbf{z})+\tau(x)
$$

and hence

$$
\mu \mathbf{u}-\left[\mathbf{K}_{n}-\nu \mathbf{I}_{n}\right] \mathbf{z}=\tau(x)-\mu \mathbf{z} .
$$

Assume $\mathbf{K}_{n}$ is partitioned in the form

$$
\mathbf{K}_{n}=\left[\begin{array}{c:c}
k_{11} & \mathbf{d}_{n-1}^{T} \\
\hdashline \mathbf{C}_{n-1} & \mathbf{A}_{n-1}
\end{array}\right]
$$

and so (2.12) can be rearranged to

$$
\mathrm{T}_{n}\left[\begin{array}{l}
\mu \\
\hdashline \bar{\eta}_{n-1}
\end{array}\right]=\tau(x)-\mu\left[\begin{array}{l}
0 \\
\hdashline \eta_{n-1}
\end{array}\right],
$$

where

$$
\mathbf{T}_{n}=\left[\begin{array}{c:c}
1 & -\mathbf{d}_{n-1}^{T} \\
\hdashline \boldsymbol{\xi}_{n-1} & \nu \mathbf{I}_{n-1}^{T}=\mathbf{A}_{n-1}
\end{array}\right]
$$


It is easily shown that $T_{n}$ is nonsingular if $\nu$ is a simple eigenvalue of $\mathbf{K}_{n}$. The stability condition in [11] will hold if we can show that $T_{n}^{-1}$ is uniformly bounded. We give the following lemma.

LEMMA 1. There exists a constant $C$, independent of $n$, and an integer $N$, such that for all $n \geqslant N,\left\|\mathbf{T}_{n}^{-1}\right\|_{\infty} \leqslant C$.

Proof. We outline the main steps of the proof.

(a) Routine manipulation shows that

$$
\left\|\mathbf{T}_{n}^{-1}\right\|_{\infty} \leqslant\left\|\mathbf{B}_{n+1}^{-1}\right\|_{\infty}
$$

where

$$
\mathbf{B}_{n+1}=\left[\begin{array}{l:c}
\nu \mathbf{I}_{n}-\mathbf{K}_{n} & \mathbf{u} \\
\hdashline \mathbf{e}_{1}^{T}- & 0
\end{array}\right],
$$

where $\mathbf{e}_{1}^{T}=[1,0, \ldots, 0]$. Thus

$$
\begin{aligned}
\mathbf{B}_{n+1} & =\nu \mathbf{I}_{n+1}-\nu\left[\begin{array}{c:c}
\nu^{-1} \mathbf{K}_{n} & -\nu^{-1} \mathbf{u} \\
\hdashline-\nu^{-1} \mathbf{e}_{1}^{T} & 1
\end{array}\right] \\
& =\nu\left(\mathbf{I}_{n+1}-\mathbf{A}_{n+1}\right), \text { say. } \\
\therefore\left\|\mathbf{T}_{n}^{-1}\right\|_{\infty} & \leqslant|\nu|^{-1}\left\|\left(\mathbf{I}_{n+1}-\mathbf{A}_{n+1}\right)^{-1}\right\|_{\infty} .
\end{aligned}
$$

(b) Define the operator $A_{n+1}: C[a, b] \times R \rightarrow C[a, b] \times R$ by

$$
A_{n+1}=\left[\begin{array}{c:c}
\nu^{-1} K_{n} & -\nu^{-1} u \\
\hdashline-\nu^{-1} l(\cdot) & 1
\end{array}\right]
$$

where $u$ and $K_{n}$ are given by (1.6) and (1.7) respectively, and

$$
l(z)=\mathbf{e}_{1}^{T} r_{n} z \quad \text { for } z \in C[a, b] .
$$

Also define the operator $A: C[a, b] \times R \rightarrow C[a, b]$ by

$$
A=\left[\begin{array}{c:c}
\lambda^{-1} K & -\lambda^{-1} x \\
\hdashline-\lambda^{-1} l(\cdot) & 1
\end{array}\right] .
$$

Now, assuming A2 and A5, $(I-A)^{-1}$ exists using Lemma 3.2 of [1]. Standard application of collectively compact operator theory gives

$$
\left\|\left(I-A_{n+1}\right)^{-1}\right\|_{\infty}<C_{1}\left(1+\left\|(I-A)^{-1}\right\|_{\infty}\|A\|_{\infty}\right)<C_{2}
$$

say, for $n \geqslant N$, and it follows that there exists a constant $C$ independent of $n$ such that

$$
\left\|\left(\mathbf{I}_{n+1}-\mathbf{A}_{n+1}\right)^{-1}\right\|_{\infty} \leqslant C .
$$

Hence, from (a), $\mathbf{T}_{n}^{-1}$ is uniformly bounded. 
Thus (2.13) will be a suitable equation on which to base an iteration scheme. We note in passing that (2.13) can be used to prove (1.10). (See [13].) The deferred correction process can now be written as

$$
\mathrm{T}_{n}\left[\begin{array}{c}
\mu^{(r)} \\
\hdashline \eta_{n-1}^{(r)}
\end{array}\right]=\delta^{p}\left(\mathcal{U}^{(r-1)}\right)-\mu^{(r-1)}\left[\begin{array}{c}
0 \\
\hdashline \eta_{n-1}^{(r-1)}
\end{array}\right], \quad r=1,2, \ldots,
$$

with

$$
\mu^{(0)}=0, \eta_{n-1}^{(0)}=\mathbf{0}
$$

The arguments of $\delta^{p}(\cdot)$ are the net functions $\left\{\mathcal{Q}^{(r)}\right\}$ which satisfy

$$
\text { (i) } \begin{aligned}
\mathscr{U}^{(r)} & \in C^{p+2}[a, b] ; \\
\text { (ii) } r_{n} \mathscr{U}^{(r)} & =\mathbf{u}+\left[0, \eta_{n-1}^{(r)}\right]^{T} .
\end{aligned}
$$

These net functions (defined below) are introduced to prove the rate of convergence result and are only of theoretical interest. Because of the nonlinear terms in (2.13) and (2.14) the theory given in [11], [16] needs to be slightly modified, although the essential ideas remain the same.

We define the net functions $\left\{\mathcal{Q}^{(r)}\right\}$ by

$$
\text { (i) } \mathscr{U}^{(0)}=u \text { defined by (1.7); }
$$

(ii) $\nu \mathscr{U}^{(r)}=K_{n}\left(r_{n} \mathscr{U}^{(r)}\right)+\delta_{s}^{p}\left(\mathcal{U}^{(r-1)}\right)-\mu^{(r-1)}\left(\mathcal{U}^{(r-1)}-\mathcal{Q}^{(0)}\right)-\mu^{(r)} \mathcal{U}^{(0)}$

for $r=1,2, \ldots$ with $\mu^{(r)}$ given by (2.14) (recall (2.8)) and $\delta_{s}^{p}(\cdot)$ given by (2.6). Condition (2.15i) follows from A3 and it is straightforward to verify (2.15ii) after recalling the derivation of (2.13) from (2.11).

We now state the main theorem.

THEOREM 2. Assume A1-A5 and let $\mu^{(r)}, \eta_{n-1}^{(r)}$ be given by (2.14). For large enough $n$, and for $2 r \leqslant p, p \geqslant 2$,

$$
\lambda-\left(\nu+\mu^{(r)}\right)=O\left(h^{2 r+2}\right)
$$

and

$$
\mathbf{x}-\left(\mathbf{u}+\left[0, \eta_{n-1}^{(r)^{T}}\right]^{T}\right)=O\left(h^{2 r+2}\right) .
$$

Proof. (a) Consider first the case $r=1$. From (1.7), (1.9), (2.8) and (2.16i) we have

$$
\nu\left(x-\mathcal{Q}^{(0)}\right)=K_{n}\left(r_{n} x-r_{n} \mathcal{Q}^{(0)}\right)+\tau(x)-\mu x .
$$

Clearly $x-\mathcal{Q}^{(0)} \in C^{p+2}[a, b]$ and since $r_{n} \mathcal{Q}^{(0)}=\mathbf{u}$ we have, using (2.2), (2.3) and (2.10),

$$
x-\mathcal{U}^{(0)}=O\left(h^{2}\right)
$$


Also from the differentiability with respect to $s$ of $K_{n}$ and $\tau(x)$ we have

$$
\left[x-\mathcal{Q}^{(0)}\right]^{(l)}=O\left(h^{2}\right), \quad l=1,2, \ldots, p+2,
$$

where $[\cdot]^{(l)}$ denotes the $l$ th derivative. Hence using (2.4)

$$
\tau\left(x-\mathcal{U}^{(0)}\right)=O\left(h^{4}\right)
$$

and, using (2.5),

$$
\tau(x)-\delta_{s}^{p}\left(\mathcal{Q}^{(0)}\right)=O\left(h^{4}\right) .
$$

From (2.13) and (2.14) we have

$$
\mathrm{T}_{n}\left[\begin{array}{c}
\mu-\mu^{(1)} \\
\hdashline \eta_{n-1}-\boldsymbol{\eta}_{n-1}^{(1)}
\end{array}\right]=\tau(x)-\delta^{p}\left(\mathcal{U}^{(0)}\right)-\mu\left[\begin{array}{c}
0 \\
\hdashline \eta_{n-1}^{-}
\end{array}\right]=O\left(h^{4}\right)
$$

using (2.10), (2.20). Clearly, since $\mathbf{T}_{n}$ is uniformly bounded,

$$
\mu-\mu^{(1)}, \eta_{n-1}-\eta_{n-1}^{(1)}=O\left(h^{4}\right)
$$

and (2.8), (2.9) give (2.17), (2.18) for $r=1$.

Now consider $x-\mathcal{Q}^{(1)}$. From (1.9) and (2.16ii) we have

$$
\begin{aligned}
\nu\left(x-\mathcal{U}^{(1)}\right)= & K_{n}\left(r_{n} x-r_{n} \mathcal{U}^{(1)}\right)+\tau(x)-\delta_{s}^{p}\left(\mathcal{Q}^{(0)}\right) \\
& -\mu x+\mu^{(1)} \mathcal{U}^{(0)}=O\left(h^{4}\right),
\end{aligned}
$$

using (2.20) and

(i) $r_{n} x-r_{n} \mathscr{Q}^{(1)}=r_{n} x-\left\{\mathbf{u}+\left[0, \eta_{n-1}^{(1)}\right]^{T}\right\}=O\left(h^{4}\right)$;

(ii) $\mu x-\mu^{(1)} \mathcal{Q}^{(0)}=\left(\mu-\mu^{(1)}\right) x+\mu^{(1)}\left(x-\mathcal{Q}^{(0)}\right)=O\left(h^{4}\right)$ since $\mu^{(1)}=O\left(h^{2}\right)$. From the differentiability of $K_{n}, \tau(\cdot)$ and $\delta_{s}^{p}(\cdot)$ we have

$$
\left[x-\mathcal{U}^{(1)}\right]^{(l)}=O\left(h^{4}\right), \quad l=1,2, \ldots, p+2,
$$

and from (2.4),

provided $p \geqslant 4$. Hence

$$
\tau\left(x-\mathcal{U}^{(1)}\right)=O\left(h^{6}\right)
$$

$$
\tau(x)-\delta_{s}^{p}\left(\mathscr{U}^{(1)}\right)=O\left(h^{6}\right)
$$

using (2.5). The results

$$
\mu-\mu^{(2)}, \eta_{n-1}-\eta_{n-1}^{(2)}=O\left(h^{6}\right)
$$

follow immediately by repeating the argument following (2.20) with $r=2$.

(b) The proof of the general result follows by induction using steps similar to those above. The important result is

$$
\tau(x)-\delta_{s}^{p}\left(U^{(r)}\right)=O\left(h^{2 r+4}\right), \quad r=0,1,2, \ldots, 2 r<p .
$$

Q.E.D.

Clearly, under assumption A3 we can only obtain estimates of $\lambda$ and $\mathbf{x}$ up to $O\left(h^{p+2}\right)$. 


\section{Discussion}

In this section we gather together various remarks about deferred correction and its application to the eigenvalue problem.

REMARK 1. A satisfactory analysis of the method of deferred correction depends on a successful treatment of the quantity $\tau(x)-\delta_{s}^{p}\left(Q^{(r)}\right)$, or equivalently $\delta_{s}^{p}\left(\mathcal{U}^{(r)}\right)-\delta_{s}^{p}\left(\mathcal{U}^{(r-1)}\right)$. (Compare (c) of the theorem in Section 4 of [16].) The key result is (2.22). The analysis of deferred correction for second kind equations in [5] fails to produce the observed rate of convergence because the result obtained for $\delta^{p}\left(\mathcal{U}^{(r-1)}\right)-\delta^{p}(x)$ is not the best possible. Theorem 4.22 of [5] states (in the notation of this paper)

$$
\left\|\mathbf{x}^{(r)}-r_{n} x\right\|_{\infty}=O\left(h^{r+2}\right)
$$

where $x$ satisfies $(2.1)$ and $x^{(r)}$ is computed from

$$
\left[\lambda \mathbf{I}_{n}-\mathbf{K}_{n}\right] \mathbf{x}^{(r)}=\mathbf{f}+\boldsymbol{\delta}^{p}\left(x^{(r-1)}\right)
$$

where $r_{n} x^{(r-1)}=\mathrm{x}^{(r-1)}$. The result (3.1) can be improved as follows. Define the functions $\mathcal{Q}^{(r)}$ by

$$
\lambda \mathcal{U}^{(0)}=K_{n}\left(r_{n} \mathcal{Q}^{(0)}\right)+f
$$

and

$$
\lambda \mathcal{Q}^{(r)}=K_{n}\left(r_{n} \mathcal{Q}^{(r)}\right)+f+\delta_{s}^{p}\left(\mathcal{Q}^{(r-1)}\right), \quad r=1,2, \ldots
$$

Then one can show that

$$
\delta_{s}^{p}\left(\mathcal{U}^{(r)}\right)-\tau(x)=O\left(h^{2 r+2}\right),
$$

provided that $2 r \leqslant p$ and hence obtain the rate of convergence result

$$
\left\|\mathrm{x}^{(r)}-r_{n} x\right\|_{\infty}=O\left(h^{2 r+2}\right) \text {. }
$$

(Compare with (3.1).)

REMARK 2. It is clear that a theorem similar to theorem 2 above could be given for the iterated deferred correction approach given by (2.14) with $\delta^{p}\left(\mathcal{U}^{(r-1)}\right)$ replaced by $\delta^{2 r}\left(\mathcal{U}^{(r-1)}\right)$. A similar modification is required in (2.16ii).

REMARK 3. Though the above analysis is given for the trapezoidal rule (recall assumption Al) the method of analysis will hold for any quadrature rule with suitable expansions for $\tau(x)$.

REMARK 4. If we replace $\tau(x)$ in $(2.7)$ by $\delta^{p}(x)$ then we obtain the matrix equation

$$
\left[\tilde{\lambda} \mathbf{I}_{n}-\mathbf{K}_{n}^{(p)}\right] \tilde{\mathbf{x}}=\mathbf{0}
$$


where $\left(\mathbf{K}_{n}^{(p)}\right)_{i j}=w_{j, p} k\left(t_{i}, t_{j}\right)$ with $w_{j p}$ being the weights of the $p$ th Gregory rule. Equation (2.14) is precisely a reformulation of (3.2) and so (3.2) could be solved directly instead of (1.4) and then (2.14). However one is left with the "a priori" choice of $p$ and some advantages of deferred correction (discussed in the next paragraph) are lost.

The method of deferred correction for integral equations has the usual advantages of such methods. Briefly the main points are:

(1) Highly accurate solutions can be obtained for small values of $n$ when the kernel is smooth. This is particularly advantageous if $k(s, t)$ is expensive to evaluate.

(2) Estimates of $\lambda$ and $\mathbf{x}$ are given by successive iterates which can be compared to give an indication of reliability.

(3) For symmetric kernels symmetry can be retained in (1.4) if the weights remain positive [5, p. 374]. Note that for some $p$ the weights $w_{j p}$ in (3.2) are not all positive and in such cases symmetry cannot be preserved.

REMARK 5. Theoretically the method works well "if $n$ is large enough". However, for computational efficiency, $n$ should be chosen to be as small as possible. Ideally we would like to be able to test whether for a given $n$ the values $\nu$, u obtained from (1.4) are good enough to ensure that the deferred correction iteration (2.14) will converge without any other checks. The following test has been incorporated in programs for deferred correction in [7] and has proved to be very reliable. Assume that the trapezoidal rule has been used to construct (1.4). From the theory of quadrature methods [12] we have that (2.3) holds provided

$$
|\lambda|>\left\|\left(1-p_{n} r_{n}\right) K\right\|_{\infty}
$$

where $p_{n}$ is a suitably chosen prolongation. If $k \in C^{2}([a, b] \times[a, b])$ then (3.3) becomes

$$
|\lambda|>h^{2}\left\|K_{s s}\right\|_{\infty} / 8
$$

where $K_{s s}$ is the integral operator with kernel $\partial^{2} k(s, t) / \partial s^{2}$. It may be possible to compute $\left\|K_{s s}\right\|_{\infty}$ exactly for certain simple kernels but more probably a numerical approximation will have to be used. The simplest test to decide whether or not $\nu$ is an "acceptable" approximation to $\lambda$ is

$$
|\nu|>h^{2}\left\|K_{s s}\right\|_{\infty} / 8
$$

and this works well in practice. A safety factor could be included in (3.4). 
ReMARK 6. Recall that assumption A4 was made to help in the explanation of the theory. In an actual computation the component of maximum modulus of $u$, say $(u)_{m}$, will have to be found and the difference $x-u$, given by (2.9), will have the form

$$
\mathbf{x}-\mathbf{u}=\left[\boldsymbol{\eta}_{1, m-1}^{T}, 0, \boldsymbol{\eta}_{2, n-m}^{T}\right]^{T},
$$

where $\eta_{1, m-1}$ is a $(m-1)$ vector and $\eta_{2, n-m}$ is an $(n-m)$ vector. Clearly the structure of $T_{n}$ will also need to be changed, as will the iteration (2.14) and the result (2.18). These changes present no problems in implementation.

In Section 4 an example is given with $m=(n+1) / 2$.

Remark 7. The theory in Section 2 above has been extended to the multiple eigenvalue case in [7]. Briefly, consider the case when $\lambda$ is an eigenvalue of $K$ of multiplicity $l$. The matrix $\mathbf{K}_{n}$ will have a cluster of $l$ eigenvalues $\nu_{i}, i=1, \ldots, l$, close to $\lambda$ [2]. Equations (1.4) and (2.7) are replaced by

$$
\mathbf{K}_{n} \mathbf{U}_{n, l}=\mathbf{U}_{n, l} \mathbf{N}_{l}
$$

and

$$
\mathbf{K}_{n} \mathbf{X}_{n, l}+\mathscr{T}\left(x_{1}, \ldots, x_{l}\right)=\mathbf{X}_{n, l} \mathbf{L}_{l}
$$

respectively, where $\mathbf{N}_{l}$ and $\mathbf{L}_{l}$ are $l \times l$ matrices, and $\mathbf{U}_{n, l}, \mathbf{X}_{n, l}$ and $\mathcal{T}\left(x_{1}, \ldots, x_{l}\right)$ are $n \times l$ matrices. Perturbation theory, similar to that described in [15] but retaining the normalisation of the eigenvectors of $\mathbf{K}_{n}$ used in Section 2 , is used to derive an equation equivalent to (2.13). Allowance can be made for the defective case.

\section{Example}

Consider the following kernel

$$
k(s, t)=\left\{\pi\left(1+(s-t)^{2}\right)\right\}^{-1} ; \quad a=-1, b=1 ;
$$

which arises from a problem in electrostatics. Clearly $k \in C^{\infty}([-1,1] \times[-1,1])$ and is ideally suited for the method of deferred correction. An exact value for the dominant eigenvalue is not known but has been estimated as $\lambda_{1}=$ .4535728641 . To illustrate the theory the errors $E_{n, r}=\left|\lambda-\left(\nu+\mu^{(r)}\right)\right|$, obtained using the method of iterated deferred correction (see Remark 2 above), are given in Table 1 below. 
TABLE 1

Values of $E_{n, r}$

\begin{tabular}{c|c|c|c}
$r \backslash n$ & 9 & 17 & 33 \\
\hline 0 & $.209 \mathrm{E}-1$ & $.522 \mathrm{E}-3$ & $.130 \mathrm{E}-3$ \\
1 & $.150 \mathrm{E}-3$ & $.849 \mathrm{E}-5$ & $.479 \mathrm{E}-6$ \\
2 & $.302 \mathrm{E}-4$ & $.737 \mathrm{E}-6$ & $.106 \mathrm{E}-7$ \\
3 & $.552 \mathrm{E}-5$ & $.756 \mathrm{E}-7$ & $.719 \mathrm{E}-9$
\end{tabular}

As expected the method produces good results. To examine the rate of convergence we give the ratios of the errors for successive values of $n$ in Table 2. The theoretical rate is $O\left(h^{2 r+2}\right)$, see (2.17), and since $h$ is being reduced by a factor of 2 each time the expected value of $E_{n, r} / E_{(2 n-1), r}$ is $2^{2 r+2}$. The numerical results, at least for small values of $r$, are in good agreement with the theory.

TABLE 2

Values of $E_{9, r} / E_{17, r}$ and $E_{17, r} / E_{33, r}$

\begin{tabular}{c|c|c|c}
$r \backslash$ & $E_{9, r} / E_{17, r}$ & $E_{17, r} / E_{33, r}$ & Expected Ratio \\
& & & $\left(=2^{2 r+2}\right)$ \\
\hline 0 & 4.0 & 4.0 & 4 \\
1 & 17.7 & 17.7 & 16 \\
2 & 41.0 & 69.5 & 64 \\
3 & 73.0 & 105.1 & 256
\end{tabular}

Numerical results for the eigenfunction corresponding to $\lambda_{1}$ again show reasonable agreement with the theory. Note that in this case $\|\mathbf{u}\|_{\infty}=(\mathbf{u})_{m}$, $m=(n+1) / 2$. (Recall Remark 6 in Section 3.) In Table 3 we give the values

$$
F_{n, r}=x\left(\frac{1}{4}\right)-\left(\mathbf{u}+\left[\boldsymbol{\eta}_{1, m-1}^{(r)^{T}}, 0, \eta_{2, n-m}^{(r)^{T}}\right]\right)_{i}^{T}, \quad i=(n+3) / 4,
$$

(recall (2.18), modified as suggested by (3.5)) and in Table 4 the ratios $F_{n, r} / F_{(2 n-1), r^{*}}$

\section{TABLE 3}

Values of $F_{n, r}$

\begin{tabular}{c|c|c|c}
$r^{\wedge}$ & 9 & 17 & 33 \\
\hline 0 & $.423 \mathrm{E}-3$ & $.103 \mathrm{E}-3$ & $.250 \mathrm{E}-3$ \\
1 & $.259 \mathrm{E}-3$ & $.205 \mathrm{E}-4$ & $.125 \mathrm{E}-5$ \\
2 & $.728 \mathrm{E}-4$ & $.608 \mathrm{E}-5$ & $.660 \mathrm{E}-7$ \\
3 & $.583 \mathrm{E}-3$ & $.297 \mathrm{E}-5$ & $.408 \mathrm{E}-8$
\end{tabular}


TABLE 4

\begin{tabular}{c|c|c|c}
\multicolumn{4}{c}{ Values of $F_{9, r} / F_{17, r}$ and $F_{17, r} / F_{33, r}$} \\
$r$ & $F_{9, r} / F_{17, r}$ & $F_{17, r} / F_{33, r}$ & Expected Ratio \\
& & & $\left(=2^{2 r+2}\right)$ \\
\hline 0 & 4.1 & 4.1 & 4 \\
1 & 12.6 & 16.4 & 16 \\
2 & 11.9 & 92.1 & 64 \\
3 & 196.2 & 727.9 & 256
\end{tabular}

References

[1] P. M. Anselone and L. B. Rall, "The solution of characteristic value-vector problems by Newton's method", Numer. Math. 11 (1968), 38-45.

[2] K. E. Atkinson, "Convergence rates for approximate eigenvalues of compact integral operators", SIAM J. Numer. Anal. 12 (1975), 213-221.

[3] K. E. Atkinson, A survey of numerical methods for the solution of Fredholm integral equations of the second kind, Society for Industrial and Applied Mathematics, (1976).

[4] C. T. H. Baker, "The deferred approach to the limit for eigenvalues of integral equations", SIAM J. Numer. Anal. 8 (1971), 1-10.

[5] C. T. H. Baker, The numerical treatment of integral equations, Clarendon Press, (1977).

[6] F. E. Chatelin, "Numerical computation of the eigenelements of linear integral equations by iterations", SIAM J. Numer. Anal. 15 (1978), 1112-1124.

[7] K. W. Chu, The improvement of approximate solutions of the integral equation eigenvalue problem (Ph. D. Thesis, University of Bath, 1980).

[8] L. Fox and E. T. Goodwin, "The numerical solution of nonsingular linear integral equations", Phil. Trans. Roy. Soc. A, 245, (1953), 501-534.

[9] W. Hackbush, "On the computation of approximate eigenvalues and eigenfunctions of elliptic operators by means of a multigrid method", SIAM J. Numer. Anal. 16, (1979), 201-215.

[10] D. F. Mayers, "Fredholm equations of first and third kinds", Chapters 11, 12 of Numerical solution of ordinary and partial differential equations, (ed. L. Fox) (Pergamon Press, 1962).

[11] V. Pereyra, "On improving an approximate solution of a functional equation by deferred corrections", Numer. Math. 8 (1966), 376-391.

[12] A. Spence, "On the convergence of the Nyström method for the integral equation eigenvalue problem", Numer. Math. 25 (1975), 57-66.

[13] A. Spence, "Error Bounds and Estimates for Eigenvalues of Integral Equations II", Numer. Math. 32 (1979), 139-146.

[14] H. J. Stetter, "The defect correction principle and discretization methods", Numer. Math. 29 (1978), 425-443.

[15] G. W. Stewart, "Error and Perturbation bounds for subspaces associated with certain eigenvalue problems", SIAM Rev. 15 (1973), 727-764. 
[16] J. M. Watt, "Convergence and stability of discretization methods for functional equations", Computer J. 11 (1968), 77-82.

[17] J. H. Wilkinson, The algebraic eigenvalue problem, (Oxford University Press, 1965).

School of Mathematics

University of Bath

Claverton Down

Bath BA2 7AY

United Kingdom 